\title{
THE SURFACE BRIGHTNESS OF OUR GALAXY AND OTHER SPIRALS
}

\author{
P. C. van der Kruit \\ Kapteyn Astronomical Institute \\ P.O. Box 800 \\ 9700 AV Groningen, The Netherlands
}

\begin{abstract}
In this review I discuss some aspects of the luminosity distributions in our Galaxy and external spiral galaxies. The major conclusions are the following: (1) the radial scale length of the luminosity distribution in the disk of our Galaxy is $5.0 \pm 0.5 \mathrm{kpc}$, (2) on this basis the Hubble constant needs to be at most $65 \pm 10 \mathrm{~km} \mathrm{~s}^{-1} \mathrm{Mpc}^{-1}$, if our Galaxy and M31 are among the largest spirals, as the Fisher-Tully relation suggests, (3) the probable Hubble type of the Galaxy is SbI-II, (4) the bi-modal distribution function of face-on, central surface brightness $\mu_{0}$ and radial scale length $h$ of spirals shows a preferred value for $\mu_{0}$ of about 22 B-mag $\operatorname{arcsec}^{2}$ and a distribution of $h$ that declines with one efolding per kpc, (5) the Galaxy is a normal, fairly large Sb galaxy, and (6) galaxies similar to our own in terms of large-scale, nonmorphological properties are NGC 891 and NGC 5033.
\end{abstract}

\section{INTRODUCTION}

In the following I will review the results of modeling the large-scale distribution of stars in the Milky Way Galaxy and compare these with the statistical distribution of surface brightness parameters of other spiral galaxies. In addition, I will illustrate how a determination of the radial scale length of the disk of the Galaxy can be used to constrain the Hubble constant, and I will look into the possibility of identifying other spirals that bear a close resemblance to our own. Unless indicated otherwise, distances quoted below are based on a Hubble constant of $75 \mathrm{~km} \mathrm{~s}^{-1} \mathrm{Mpc}^{-1}$. In the Galaxy I use a distance of the sun to the center of $8.5 \mathrm{kpc}$.

\section{PHOTOMETRIC COMPONENTS IN GALAXIES}

The surface brightness of external spiral galaxies can be represented by two components, and on this basis the observed surface brightness of the Milky Way is likewise represented by the same components. The first is the bulge or spheroid, which is centrally concentrated and only moderately flattened. The projected surface brightness follows the empirical " $R$ "-law" defined first by de Vaucouleurs (1948). It appears to be able to represent actual profiles in bulges and elliptical galaxies over a very wide range in surface brightness. Its equation is

$$
\log \left[I(R) / I_{\mathrm{e}}\right]=-3.3307\left[\left(R / R_{\mathrm{e}}\right)^{1 / 4}-1\right] .
$$

Here $R_{\mathrm{e}}$ is the effective radius that encloses half the total light and $I_{\mathrm{e}}$ the surface brightness at that radius. The central surface brightness (in mag $\operatorname{arcsec}^{-2}$ ) then becomes $\mu_{0}=\mu_{e}+8.3268$. The corresponding total luminosity is

$$
L=7.215 \pi I_{\mathrm{e}} R_{\mathrm{e}}^{2}(b / a),
$$

where $b / a$ is the apparent axis ratio. 
The description of the radial surface brightness distribution in disks followed that of bulges and ellipticals by about three decades. This is an understandable result of the much fainter surface brightness of the disks. There is one simple law for the radial distribution of surface brightness, namely that of the exponential disk:

$$
I(R)=I_{0} \exp (-R / h) \text {. }
$$

The parameter $h$ is usually referred to as the disk scale length. The integrated magnitude is

$$
L=2 \pi h^{2} I_{0} \text {. }
$$

The exponential law was first described in an unpublished Harvard thesis based on observations of M33. A short report is available in Patterson (1940), and a plot of the data appears in the extensive description of light distributions in galaxies by de Vaucouleurs (1959, his Figure 10). The first detailed work was performed by de Vaucouleurs in a series of papers in the late 1950s that involved the suitable members of the Local Group: LMC, M31, and M33. This fundamental work established once and for all that exponentials are the rule and occur in $\mathrm{Sb}, \mathrm{Sc}$, and irregular galaxies.

Many studies have in the mean time confirmed the universal applicability of the exponential as a fitting function. It should, however, be noted that in all galaxies for which an exponential has been fitted to the disk surface brightness profile, there are important, nonlocal deviations from the fit at a level of a few tenths of a magnitude, while often the fit can be performed over a few magnitudes' (magnitudes are roughly e-foldings) interval in the first place (e.g., van der Kruit 1987a). I will discuss the distribution of the central surface brightness and the scale length below in some detail (see also van der Kruit $1987 a$, where also a possible model for the origin of exponential disks is described); however, here I already note that Freeman (1970) found that large spiral galaxies appear to display a remarkably small range in extrapolated central (face-on) surface brightness, namely $21.65 \pm 0.30 \mathrm{~B}-\mathrm{mag} \operatorname{arcsec}^{-2}$.

Disks do have a finite thickness. The three-dimensional structure therefore requires a description of the vertical distribution. Van der Kruit and Searle $(1981 a, b)$ proposed to use the formula for the (locally) isothermal sheet for this, so that the full description becomes

$$
L(R, z)=L(0,0) \exp (-R / h) \operatorname{sech}^{2}\left(z / z_{0}\right) .
$$

The face-on surface brightness follows from

$$
I(R)=2 z_{0} L(R, 0),
$$

and the vertical scale parameter $z_{0}$ relates to the surface density $I(R)(M / L)$ and the velocity dispersion $\left\langle V_{z}^{2}\right\rangle^{1 / 2}$ as

$$
\left\langle V_{z}^{2}\right\rangle=\pi G I(R) z_{0}(M / L) .
$$

The reason for choosing the isothermal sheet was the observation in the solar neighborhood (e.g., Wielen 1977) that stars that have ages above a few Gyr all have roughly the same velocity dispersion, so that the mix of disk stars should be dominated-at least moderately far from the plane-by essentially a single stellar population with a single velocity dispersion. This component is the "old disk population" in terms of the population scheme put forward at the famous "Vatican Symposium" on stellar populations in 1957. Furthermore, earlier observations (e.g., van der Kruit 1979) had indicated that disks have roughly exponential $z$-profiles with the scale parameter independent of galactocentric distance. The latter agrees with the isothermal disk, because for large $z\left(z / z_{0}>>1\right)$ :

$$
\operatorname{sech}^{2}\left(z / z_{0}\right)=4 \exp \left(-2 z / z_{0}\right) \text {. }
$$


Actual fits of the proposed three-dimensional distribution to photometry of edge-on galaxies with little or no bulge confirmed the applicability and, in particular, the remarkable property that $z_{0}$ is constant with $R$. It should be stressed that the description refers only to the old disk population and ignores effects from young population I stars and dust.

It was also found earlier that disks have rather sharp outer edges or truncations (van der Kruit 1979). From a sample of seven edge-on spirals, van der Kruit and Searle (1982a) found that this radius $R_{\max }$ occurs at $4.2 \pm 0.6$ radial scale lengths $h$. This was not known from luminosity profiles of inclined or face-on systems, and van der Kruit (1988) has proposed that this is the result of relatively small deviations from circular symmetry in the stellar disks. These deviations correspond to variations in $R_{\max }$ of order $10 \%$ with azimuthal direction. As a result, radial profiles obtained by azimuthal averaging of the light distribution fail to show the sharp declines. Beyond the "truncation radius" the radial e-folding of the light distribution drops to values generally less than $1 \mathrm{kpc}$.

The general inference that can be drawn is that the light distributions in spiral galaxies can apparently be described very well with only two basic components: a bulge and a disk. These two basic components have discretely different flattenings and kinematics and may point to two discrete epochs of star formation, one before and one after collapse and virialization of the protogalaxy (see van der Kruit and Searle 1982b). In the bulge we find evidence for color gradients, which are interpreted as abundance gradients, and this is a feature that appears to be typical for bulges. Disks, on the other hand, are in general uniform in color with galactocentric distance.

There is now quite some evidence from star counts in the Galaxy that seems to point to a third component, intermediate between disk and halo in flattening, metallicity, and kinematics, sometimes referred to as the "thick disk" (see Gilmore, Wyse, and Kuijken 1989). Detailed analysis of the photometric data of NGC 891 (van der Kruit 1984) shows that the bulge surface brightness distribution can equally well be represented by a bulge with somewhat changing axis ratio or a combination of a bulge with constant axis ratio and a component rather similar to the "thick disk" proposed for our Galaxy. On the other hand, we find no evidence whatsoever of such a component in NGC 7814 (van der Kruit and Searle 1982b). Furthermore, in our Galaxy the "thick disk" makes up only about $10 \%$ or so of the disk light, and if associated with disk formation, it can equally well be seen as a subcomponent of the disk population, which after all goes down at the other side of the range of flattenings to a thickness of about $100 \mathrm{pc}$ for extreme Population I.

\section{SURFACE PHOTOMETRY OF OUR GALAXY}

Surface photometry of the Galaxy is very difficult because of the low surface brightness, except maybe in the galactic plane or the brighter parts of the bulge, and because it is not easy to obtain photometric stability over the very large angular scales involved. From the earth, furthermore, one has to worry about the zodiacal light, at least for higher galactic latitudes, where interesting information on large-scale Galactic structure can be obtained due to absence of absorption.

Now, fortunately, the inverse problem occurs for researchers of the zodiacal light itself, for whom the galactic background light is a nuisance. Therefore, the Pioneer 10 (and 11) spacecraft, which were sent to Jupiter and were equipped with a wide-field mapping capability in the optical, were used to study this matter (see the review by Toller in this volume). This resulted in two maps of the sky in the red and the blue with an angular resolution of $2^{\circ}$. After 
smoothing to an angular resolution of $8^{\circ}$ (which is sufficient to study Galactic large-scale structure), the maps in Figure 1 result, presented there as isophotes maps in mag $\operatorname{arcsec}^{-2}$.

Positional data by themselves cannot, in principle, be used to derive length scales. It turns out that the Pioneer data can be used to constrain the ratio of radial scale length $h$ to
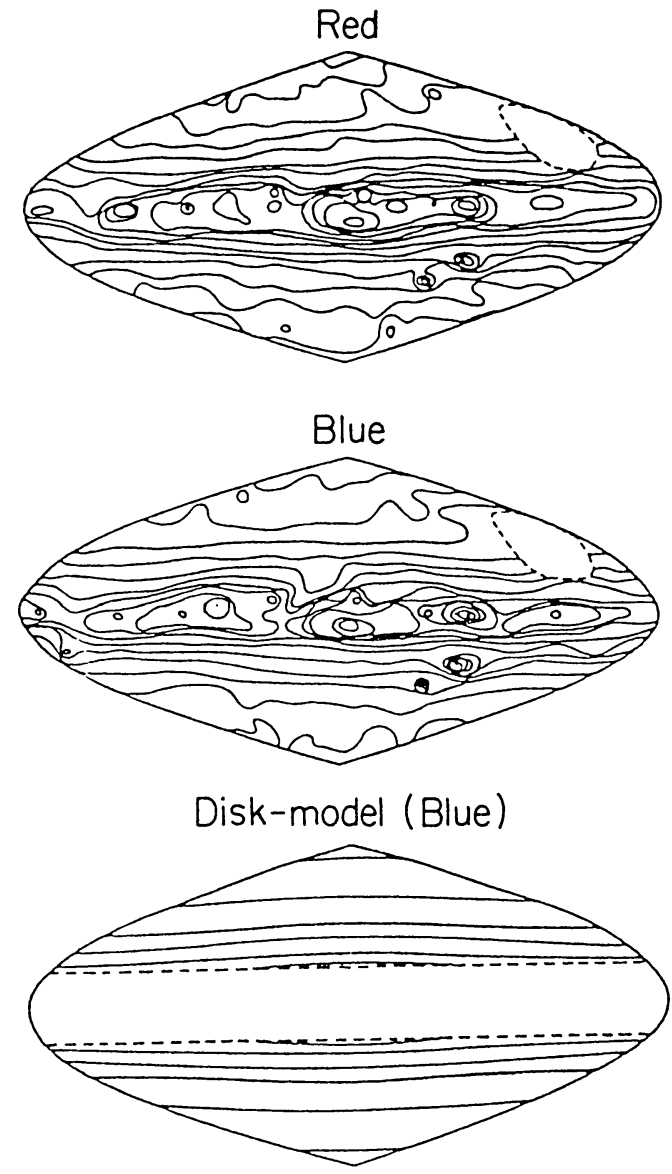

Figure 1. Isophote maps of the distribution of surface brightness from the integrated starlight from our Galaxy, as derived by Pioneer 10 on its way to and beyond Jupiter. Stars brighter than $m_{\mathrm{V}}=6.5$ have been removed. The direction toward the galactic center is in the middle of the picture. The angular resolution is $8^{\circ}$ and the contour interval is $0.25 \mathrm{mag}$. In the red band, the faintest thick contour corresponds to $24 \mathrm{~V}$-mag $\operatorname{arcsec}^{-2}$ and in the blue band to $24 \mathrm{~B}-\mathrm{mag} \operatorname{arcsec}^{-2}$. The missing part is the general direction of the sun as seen from Jupiter around Pioneer 10 encounter. The galactic bulge, Carina spiral arm region, and the Magellanic Clouds are prominent. From van der Kruit (1986). vertical scale parameter (van der Kruit 1986). Estimates for $z_{0}$ can be obtained from star count studies towards the galactic poles. Of course, the areas of high extinction $(\mathrm{E}(\mathrm{B}-\mathrm{V})>0.09 \mathrm{mag})$ have been excluded, restricting the analysis essentially to latitudes above $\sim 20^{\circ}$. The exclusion of low latitudes is the reason that the ratio of scale length to the solar galactocentric radius cannot be extracted from the data. It turns out also that the bulge is too faint to contribute sufficiently for a detailed study. The method of analysis for the Pioneer data (van der Kruit 1986) has been to use the Bahcall and Soniera (1984) computer code to derive surface brightnesses for various combinations of length scales.

The major result is a definite value for the ratio $h / z_{0}$ of $8.5 \pm 1.3$. Using the value for the exponential old disk scale height in the solar neighborhood from Gilmore and Reid (1983) and others of $350 \pm 50 \mathrm{pc}$, which equals $0.5 z_{0}$, results in a radial scale length $h$ of the disk of $5.5 \pm 1.0 \mathrm{kpc}$. There are various other, independent arguments that can be used to derive values for $h$, and I have concluded from a detailed discussion of these that the best value is $5.0 \pm 0.5 \mathrm{kpc}$ (van der Kruit 1987b). Other results of the Pioneer 10 data are the central surface brightness of the Galactic disk (now the total disk and not the old disk population only), inferred from the surface brightness at the galactic poles and this value for the scale length, of $22.1 \pm 0.3 \mathrm{~B}$ mag $\operatorname{arcsec}^{-2}$, and the color index of the disk at the solar position of $(B-V)=0.84 \pm$ 0.15 . The latter is interestingly red and may indicate that the contribution from young population I to the surface brightness is not the major one. It may be atypical for the disk in general, although $\mathrm{Sb}$ galaxies do have disks with similar colors. The uncertainty of $0.15 \mathrm{mag}$ is large, but can in 
principle be improved using the unreduced data from Pioneer 10 and 11 . The total luminosity of the disk is $(1.8 \pm 0.3) \times 10^{10} L_{\odot}$. For the old disk, the value of $L(0,0)$ is about $5 \times 10^{-2} \mathrm{pc}^{-3}$ and the total luminosity is about $1 \times 10^{10} L_{\odot}$.

The value for $R_{\max }$ of the disk of the Galaxy is more difficult to derive. Young stars are observed in the anticenter direction to galactocentric distances of at least $22 \mathrm{kpc}$ (Chromey 1979), while a similar distance is found for $\mathrm{H}$ II regions. This gives an estimate of $20-25 \mathrm{kpc}$, in agreement with the observed ratio's $R_{\max } / h$ of $4.2 \pm 0.6$ in external edge-on galaxies.

Surface photometry of the bulge has been attempted photoelectrically by de Vaucouleurs and Pence (1978) and has been successful in the brighter parts at low latitudes. They compared the projected surface brightness on the sky to that of an $R^{1 / 4}$ bulge and came up with the values $R_{\mathrm{e}}=2.7 \mathrm{kpc}$ and $\mu_{0}=15.1 \mathrm{~B}-\mathrm{mag} \operatorname{arcsec}^{-2}$. From comparison with external galaxies, we can estimate a value for the flattening from the kinematics, using the relation between $V_{\mathrm{m}} / \sigma$ and the flattening, where $V_{\mathrm{m}}$ is the maximum rotational velocity of the bulge and $\sigma$ the central velocity dispersion (Kormendy and Illingworth 1982). Current best values are $V_{\mathrm{m}}=60 \pm 30$ $\mathrm{km} \mathrm{s}^{-1}$ and $\sigma=110 \pm 10 \mathrm{~km} \mathrm{~s}^{-1}$ (e.g., Freeman 1987) and lead to $b / a=0.7 \pm 0.15$. The total luminosity then is about $3 \times 10^{9} L_{\odot}$. The old disk population contains about $80 \%$ of the total

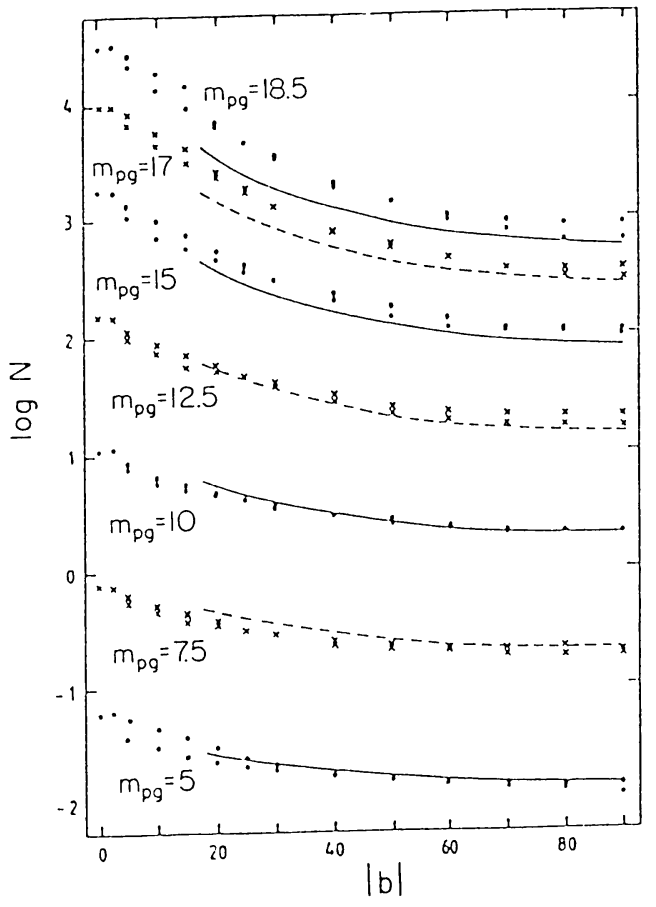

Figure 2. Comparison of the Kapteyn star counts in the selected areas reported in van Rhijn (1925) with the model of the Galaxy with the parameters derived in this section. The two values at each position come from positive and negative latitudes. From van der Kruit (1986). light in old stars, comparable to the number for NGC 891.

At this stage I want to make two remarks. The first is that the photometric parameters of our Galaxy are rather similar to those of NGC 891, which is classified as Sb. This similarity is discussed more extensively in van der Kruit (1984). The scale length of the disk of M31 is slightly larger, namely $6.0 \pm 0.5 \mathrm{kpc}$ (Walterbos and Kennicutt 1987). The second point is that we can use these data to constrain the Hubble constant by comparing these values with those for the largest spirals in the Virgo cluster (van der Kruit 1986; photometry by Watanabe 1983). The six largest $\mathrm{Sb}$ galaxies in the Virgo cluster have a scale length of $52 \pm 5^{\prime \prime}$ and the five largest Sc galaxies $50 \pm 5^{\prime \prime}$. If our Galaxy is comparable to these systems, the distance to the Virgo cluster would be $20 \pm 3$ $\mathrm{Mpc}^{-1}$ (from Sb galaxies) or $21 \pm 3 \mathrm{Mpc}$ (Sc galaxies), using $h=5.0 \pm 0.5 \mathrm{kpc}$. The scale length of M31 leads to $24 \pm 3$ Mpc. For a distance of $22 \pm 4 \mathrm{Mpc}$ with $V_{\text {rad }}($ Virgo $)=1000 \pm 50 \mathrm{~km} \mathrm{~s}^{-1}$ and a Local Group infall of $330 \pm 40 \mathrm{~km} \mathrm{~s}^{-1}$, this results in a Hubble constant $H_{0}$ of $65 \pm 10$ $\mathrm{km} \mathrm{s}^{-1} \mathrm{Mpc}^{-1}$. Comparing our Galaxy and M31 with galaxies that have smaller scale lengths results in smaller values for $H_{0}$, so the value given here should be seen as an 
upper limit. On the other hand, the Fisher-Tully relation between rotational velocity and integrated magnitude suggests that our Galaxy and M31 are among the largest spirals. We see already that these two galaxies in the Local Group probably rank among the largest spirals in the Local Supercluster. A Hubble constant of $100 \mathrm{~km} \mathrm{~s}^{-1} \mathrm{Mpc}^{-1}$ makes these probably the two largest in this volume, a very unlikely and unsatisfactory result.

It is an interesting fact that the surface brightness of the background integrated starlight is almost entirely (more than $99 \%$ ) provided by the stars brighter than magnitude 20 or so. In fact, before the Pioneer measurements this was the way in which it was estimated (e.g., Roach and Megill 1961), using the extensive work earlier this century on the Plan of Selected Areas (e.g., van Rhijn 1925). The difficulty was, however, that the old magnitude scales were unreliable. This can be checked by calculating the star counts from a Bahcall and Soniera-type model of the stellar distribution in the Galaxy, using the parameters for $h, z_{0}$, and the local disk luminosity function as given above in agreement with the Pioneer surface brightness. In Figure 2 various counts predicted from the model are compared with the ones given by van Rhijn (1925) as a function of latitude at a number of magnitudes. From this figure it is obvious that the scales go systematically wrong at fainter magnitudes, in the sense that the estimated magnitudes by van Rhijn are too bright. It is true, however that the counts are very

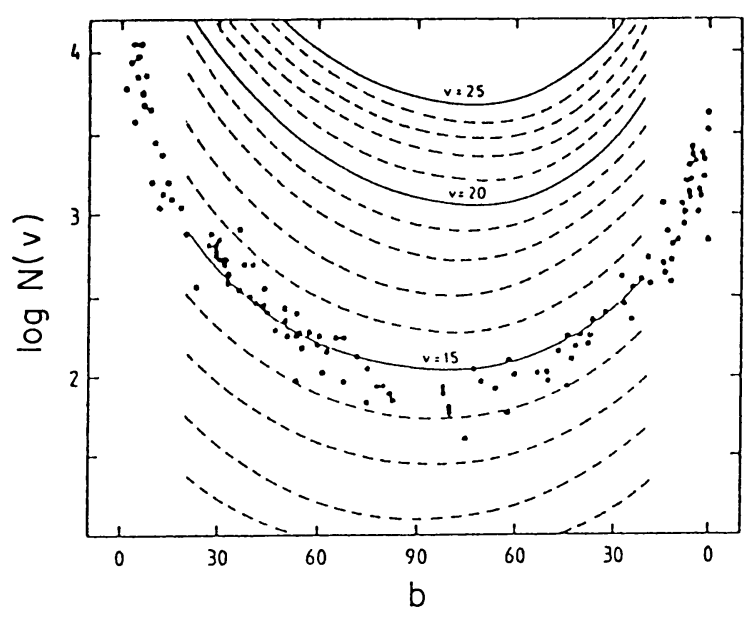

Figure 3. Comparison of the star counts made by Herschel (1785) with predictions from the Galaxy model with the parameters in this section. This great circle crosses the galactic plane at longitudes about $45^{\circ}$ and $225^{\circ}$ and misses the poles by only about $5^{\circ}$. Herschel counted stars to a limiting magnitude $V$ of about 15. (From van der Kruit 1986). consistent as a guide to relative variations that result from the stellar distribution in space.

It is also of interest to compare the model for the Galaxy with the counts that Herschel made two centuries ago. The necessary information can be found in the paper by Herschel (1785), where he publishes his famous cross-cut, of the "Sidereal System." In his analysis he assumed that his telescope was able to see all the way to the edges of the Sidereal System and that therefore the number of stars seen in a particular part of the sky could be used to calculate the distance of this edge in that direction. The procedure, how to work back from this cross-cut, is described in more detail in van der Kruit (1986). The result is given in Figure 3, where we can see that Herschel observed consistently to a limiting magnitude $V$ of about 15 . This is a remarkable achievement. 


\section{THE DISTRIBUTION OF DISK CENTRAL SURFACE BRIGHTNESS AND SCALE LENGTH IN SPIRAL GALAXIES}

In a classical study, Freeman (1970) used published surface photometry of disk galaxies to demonstrate an unexpected effect. For the majority of disks, it turned out that the extrapolated, face-on, central surface brightness $\mu_{0}$ fell in the surprisingly small range of $21.67 \pm 0.30 \mathrm{~B}$ mag $\operatorname{arcsec}^{-2}$. Exceptions were some S0s on the bright side and dwarf galaxies on the faint side. Immediately it was suspected that this constituted a selection effect, such as the one pointed out earlier by, e.g., Arp (1965). The effect is, in a few words, that for galaxies to be selected as suitable candidates for surface photometry, their central surface brightness had to fall within a limited range. Fainter surface brightnesses generally result in smaller angular diameters of the observable extent, and such galaxies are already difficult to see against the sky background. Brighter central surface brightnesses occur in systems with small luminosity scale lengths, and such galaxies appear starlike.

The problem with Freeman's result is that its significance could not be evaluated well because of the fact that the sample is not a statistically complete one, and indeed such a sample did not exist at that time. Disney (1976) was the first to investigate the effects of sample selection in a quantitative manner to see whether Freeman's result could follow from observational bias. He concluded that this could indeed be the case, and for realistic values of the sky background he could even produce Freeman's number. Furthermore, his analysis also predicted the equivalent for elliptical galaxies ("Fish's law") and the difference of about six magnitudes between the mean central surface brightness for ellipticals with an $R^{1 / 4}$ luminosity distribution and exponential disks. This concept was extended in more detail by Disney and Phillipps (1983).

Suppose that a galaxy has a luminosity law

$$
L(R)=L(0) \exp -(R / h)^{1 / b},
$$

where $b=1$ corresponds to the exponential disk and $b=4$ to the $R^{1 / 4}-1$ aw. The integrated luminosity then becomes

$$
L_{\text {tot }}=(2 b) ! \pi L(0) h^{2} \text {. }
$$

If galaxies are selected to have a diameter at the isophote $\mu_{\text {lim }}$ larger than $r_{\text {lim }}$ on the sky, it is possible for each galaxy to calculate the maximum distance $d$ out to which it will be included in the sample as

$$
d=(0.4 \ln 10)^{b}[\pi(2 b) !]^{-1 / 2}\left[\left(\mu_{\lim }-\mu_{0}\right)^{b} / r_{\lim }\right] \operatorname{dex}\left[0.2\left(\mu_{0}-M+5\right)\right] .
$$

Here again $\mu$ and $M$ are surface brightness and luminosity expressed in magnitudes. This equation is evaluated for a face-on system so that for an inclined disk, e.g., $\mu_{0}$ is the observed central surface brightness (uncorrected for inclination). This distance $d$ has a maximum as a function of $\mu_{0}$ that occurs for each value of $r_{\text {lim }}$ or $M$ at

$$
\mu_{0}=\mu_{\lim }-b(0.2 \ln 10)^{-1} \text {. }
$$

So for each galaxy we can calculate a volume (4/3) $\pi d^{3}$ within which it enters the catalogue or correspondingly a visibility $d^{3}$. In an unbiased sample and assuming uniform space densities, the number of galaxies with a particular value of $\mu_{0}$ will be present proportional to this visibility. Disney's original argument then was the following. Samples such as were studied by Freeman have been selected according to angular diameter and possibly integrated magnitude. If we select galaxies exceeding a certain diameter limit at an isophote 
$\mu_{\text {lim }}$ of about 24 B-mag $\operatorname{arcsec}^{-2}$ (which is a likely value in practice), it them follows that $d^{3}$ has a peak at 21.8 and $15.3 \mathrm{~B}-\mathrm{mag} \operatorname{arcsec}^{-2}$ for $b=1$ and $b=4$, respectively. The values now are very similar to those found by Freeman (21.6 \pm 0.3$)$ and Fish $(14.8 \pm 0.9)$, and consequently Disney has argued that this observational bias has actually produced these "laws."

Before discussing "Freeman's law" further, two points should be made: (1) it has been known from the start that dwarfs with fainter values of $\mu_{0}$ occur and their existence is therefore not an issue (what is an issue is whether or not these occur in such numbers so as to make a major contribution to the cosmic luminosity density). The early ones known were Local Group members recognized as conglomerations of faint stars and therefore selected in a completely independent way. (2) A number of studies have followed Freeman's, finding essentially the same result (although the dispersion tended to be larger). However, all of these were again based on samples that were not complete to well-defined selection criteria and, consequently, the resulting distributions could not be corrected for observational bias. The very extensive study by Grosbol (1985) is also not complete in any statistical sense.

An attempt to provide information based on statistically complete samples was reported in van der Kruit (1987a). Here galaxies were selected from background fields on deep IIIa-J Schmidt plates (providing J-magnitudes that are close to B), and after scanning the selection was made quantitative as follows: all disk galaxies (with some inclination and morphological type restrictions) that have a major axis diameter at the isophote of $26.5 \mathrm{~J}-\mathrm{mag} \operatorname{arcsec}^{-2}$ in excess of 2' were included. As it turned out-in agreement with the remarks above-this consisted of precisely all galaxies that would be selected with a similar angular size limit in the UGC. This dataset, consisting of 51 galaxies, confirmed Freeman's result for nondwarf galaxies (Sc or earlier), namely $\mu_{0}=21.52 \pm 0.39 \mathrm{mag} \mathrm{arcsec}^{-2}$ (roughly B-band). Dwarfs, which are of morphological type later than Sc and which turn out to be small in physical size as well when redshifts were available, are fainter and have $\mu_{0}=22.61 \pm 0.47 \mathrm{mag} \operatorname{arcsec}^{-2}$. Furthermore, selection as discussed above with the values for the limiting surface brightness $\mu_{\text {lim }}$ appropriate to the present sample predicted that a peak should have occurred at a considerably fainter surface brightness than estimated above, since these deep plates provided a different value for $\mu_{\text {lim. }}$. So nondwarf galaxies do have a relatively narrow distribution of $\mu_{0}$, and this is not the result of selection effects. It is interesting to note that in terms of space densities, dwarfs dominate by a large factor, but the dwarfs provide only about one-quarter of the cosmic luminosity density.

A major question is what happens at different wavelengths where absorption effects and contributions from young populations are entirely different. S. Westerhof and van der Kruit (unpublished) have repeated the analysis just described on IIIa-F plates of the same fields, as far as these existed. This gives a photometric band that is between standard $\mathrm{V}$ and $\mathrm{R}$, such that $(\mathrm{J}-\mathrm{F})=1.25(\mathrm{~B}-\mathrm{V})$, where the J-band used above is very close to $\mathrm{B}$. The means and the dispersions are the following.

\begin{tabular}{lcc} 
& J & F \\
\hline 33 nondwarfs & $21.54 \pm 0.39$ & $20.63 \pm 0.49$ \\
14 dwarfs & $22.52 \pm 0.32$ & $21.99 \pm 0.44$ \\
\hline
\end{tabular}

The ratio of the $\mathrm{J}$ - and F-scale lengths is $1.07 \pm 0.13$, so there is no significant change in $h$ with wavelength and thus again no systematic evidence for radial color gradients in disks. The distributions just given are marginally narrower in $\mathrm{J}$, but the effect is not significant. If absorption by dust and contributions of young populations are significant, one would actually have expected the F-distributions to be narrower. In agreement with the bluer colors of the dwarfs, 
the means separate, indeed, by going from $J$ to $F$.

One can also determine the distribution of scale lengths, again correcting for the known selection effects. These scale lengths exist from smaller than $1 \mathrm{kpc}$ up to $7 \mathrm{kpc}$ and the frequency distribution is, roughly speaking, exponential with an e-folding of about $1 \mathrm{kpc}$. This shows the overwhelming number density of dwarf systems (in a physical sense) and the rareness in space of large spirals like our own. On the other hand, it also follows from the data that about $10 \%$ of all disk stars in the universe still occur in disks with scale lengths larger than $4 \mathrm{kpc}$, and this is not an improbably low number.

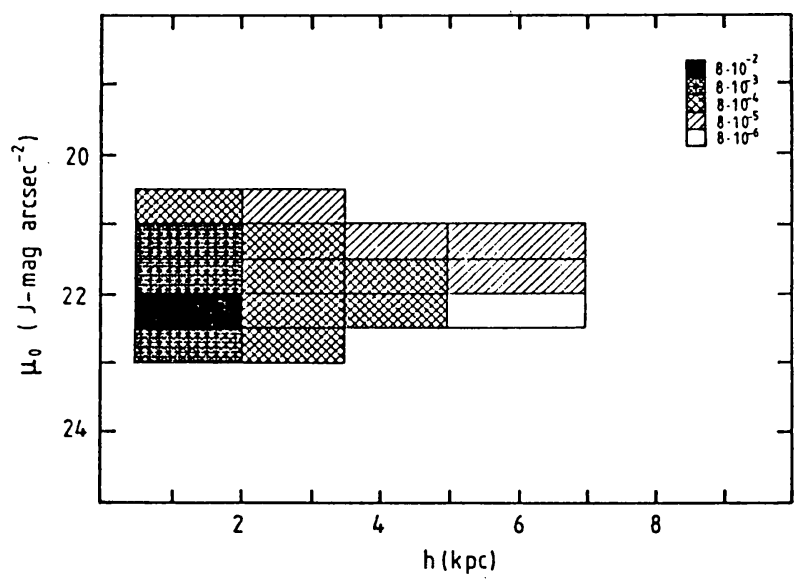

It is also possible to calculate the bivariate distribution function of $\mu_{0}$ and $h$ from such samples, although one now does require large numbers. This was done in a preliminary way for the sample above in van der Kruit $(1987 a)$, and the result is shown in Figure 4. From this it can be seen that we live in a galaxy that fits in well with the distribution observed in the universe and that appears to be one of the larger ones.

Figure 4. The bivariate distribution function of central surface brightness and radial scale length of disks in spiral galaxies. Note that the shading changes at volume densities that differ by a factor ten. From van der Kruit (1987).

\section{DISK SCALE LENGTH AND THE HUBBLE TYPE OF THE GALAXY}

An important question is what Hubble type applies to the Galaxy. The answer is to some extent dependent on what we think the disk scale length is, so that topic is discussed here in somewhat more detail than earlier. As presented in more detail above, the surface brightness of the Galaxy as measured with Pioneer 10 shows a disk scale length of $5.5 \pm 1.0 \mathrm{kpc}$. Now we look at further evidence to derive a best value.

It is important to realize that the value of $3.5 \mathrm{kpc}$ that is often seen in the literature is essentially based on a single argument by de Vaucouleurs (1979). He takes the value for the local disk surface brightness from star counts in the polar caps, assuming that the central value is Freeman's canonical one and a distance to the Galactic center. He uses also the distribution of total gas within the Sun; in some late-type galaxies, the CO distribution follows that of the blue light. However, the Galaxy shares with M31 and NGC 891 a central depression, and there is no supporting evidence that this behavior also applies to such galaxies. Finally, he uses the surface density of $\mathrm{H} \mathrm{I}$ beyond the solar circle. There are two problems there. First, the derived surface density from observations depends very sensitively on the assumed rotation curve and varies significantly between various publications. Second, in external galaxies the scale length of the $\mathrm{H} \mathrm{I}$ is different from that of the light. From the sample of Wevers, van der Kruit, and Allen (1986), the ratio of $\mathrm{H} \mathrm{I}$ to blue scale length is $1.85 \pm 0.35$. 
With the current best value for the central surface brightness and its scatter (see above) and the Pioneer value for the local surface brightness, it would follow that $h=4.2 \pm 1.0 \mathrm{kpc}$, if $R_{0}=8.5 \pm 1.0 \mathrm{kpc}$. The outer H I cannot be used well; Blitz, Fich, and Kulkarni (1983) show an $\mathrm{H}$ I distribution that is flat out to 16 or $20 \mathrm{kpc}$, and no reliable value for $h$ can be extracted with this method.

A useful estimate comes from the observation (see above) that young stars are seen in the anticenter direction out to at least $22 \mathrm{kpc}$. This puts $R_{\max }$ at $20-25 \mathrm{kpc}$. For external edge-on galaxies, van der Kruit and Searle $(1982 a)$ find $h / R_{\max }$, and it would follow that $h=5.4 \pm$ $1.0 \mathrm{kpc}$.

There are also estimates possible from stellar dynamics. Lewis (1986; see also Lewis and Freeman 1989) has measured the velocity dispersion of old disk giants as a function of galactocentric radius and find these to show an e-folding of $8.7 \pm 0.6 \mathrm{kpc}$. If, as discussed in detail elsewhere (van der Kruit and Freeman 1986), this velocity dispersion falls with an efolding twice that of the stars, then we expect $h=4.4 \pm 0.3 \mathrm{kpc}$.

A final method involves the asymmetric drift. The relevant equation is

$$
\partial \ln <V_{\mathrm{R}}^{2}>/ \partial R+\partial \ln \rho / \partial R=\left(V_{\mathrm{t}}^{2}-V_{\mathrm{rot}}^{2}\right) / R<V_{\mathrm{R}}^{2}>-[1-B /(B-A)] / R .
$$

In earlier times the first term on the left was set equal to zero. However, we now know that it is not negligible at all (as determined in both our Galaxy [Lewis's data described above] and external spirals, van der Kruit and Freeman 1986) and is most likely equal to $-1 / h$, i.e., the same as the second term. Traditionally, local density gradients are thus underestimated by a factor of 2 with this method. Using this and usual values for the local Oort constants leads, with Plaut's (1965) data for old disk variables, to $h=4.3-5.8 \mathrm{kpc}$.

From this discussion I conclude that there is ample support for a value for $h$ as indicated by the Pioneer photometry and none for the old value of $3.5 \mathrm{kpc}$. The best value for $h$ is 5.0 $\pm 0.5 \mathrm{kpc}$.

Classical ways to determine the morphological type of the Galaxy have involved arguments concerning the luminosity of the bulge, the spiral structure as determined from $\mathrm{H}$ I studies or the distribution of $\mathrm{H}$ II regions, and the local disk surface brightness. De Vaucouleurs has used many such arguments over the years and concluded on this basis that the classification is $\mathrm{SAB}(\mathrm{rs}) \mathrm{bc}$ (de Vaucouleurs and Pence 1978). In all subdivisions in this scheme, the final classification is intermediate between two designations, and the suspicion is that this indicates uncertainty rather than precision.

Color is known to correlate with Hubble type. For the Galaxy we unfortunately only know the color of the local disk, and that with a large uncertainty, namely (B-V) $=0.85 \pm$ 0.15. This is determined from the color of the galactic background starlight at high latitudes and is not necessarily typical for the disk as a whole. It would indicate a type of about $\mathrm{Sb}$, but we are probably living in a region with relatively low current star formation.

Another property that can in principle be used is the gas content. Wevers (1984) has for this purpose defined a parameter, which is the logarithm of the ratio of surface brightness in the B-band to the H I surface density, both evaluated at three optical scale lengths. For the Galaxy this property comes out as $0.5 \pm 0.3$ in solar units (van der Kruit 1986). This is similar to $\mathrm{Sb}$ galaxies (it is negative for Sc galaxies) and therefore points to such a classification.

One of the major criteria for the Hubble classification is the bulge-to-disk ratio. Quantitative determinations of this parameter show indeed a correlation as expected, but there is a large scatter within each type. The actual number gives Sbc as the most probable type, although the whole range from Sab to Scd is consistent (van der Kruit 1987b).

The most compelling argument appears to come from the CO distribution. Young (1987) has reviewed the observed radial distributions. Sc galaxies show a central peak in the CO intensities, while at least 40 (improved resolution may increase this number) of the $\mathrm{Sa}$ and $\mathrm{Sb}$ galaxies have a central hole. In this respect the Galaxy is similar to $\mathrm{Sb}$ galaxies, while at present no Sc galaxy is known, which in its CO distribution resembles the Galaxy. This 
observation seems to point rather decisively to $\mathrm{Sb}$.

Finally, we may try to find galaxies that in their properties closely resemble the Galaxy and then look at their classification. M31 (SbI-II) is similar in many respects (including the central hole in the $\mathrm{CO}$ distribution), but it has a lower current rate of star formation. This may well be within the range encountered within any particular type. A galaxy that is in many respects very similar to the Galaxy is NGC 891 (van der Kruit 1984). Not only are the photometric parameters of bulge and disk of the two systems very similar, but also the $\mathrm{CO}$ and $\mathrm{H} \mathrm{I}$ distributions and number of globular clusters are very closely the same. Both systems are seen edge-on. Indeed, many illustrations comparing photographs of NGC 891 and of the Milky Way with super-wide-angle cameras have been published, and the strong resemblance has been stressed often (e.g., King 1976). NGC 891 is invariably classified Sb.

On this basis I conclude that the most likely Hubble type of the Galaxy is $\mathrm{Sb}$, where the strongest evidence comes from the $\mathrm{CO}$ distribution, the gas content, and the strong resemblance to NGC 891. It is doubtful that we will ever be able to do better, because no quantitative observable correlates so strongly with morphological type so as to allow the exclusion of all but one classification bin. Sc is almost certainly ruled out, but Sbc is not.

The luminosity class is more difficult to determine, but a comparison to the Virgo cluster galaxies can be made. For this we look at the photometry of Watanabe (1983) in the $6^{\circ}$ core of the cluster, combined with Sandage-Tammann classifications. For $\mathrm{Sc}$ and $\mathrm{Sb}$ galaxies, it is the case that all six with class I or I-II have disk scale lengths larger than $4 \mathrm{kpc}$, while for the 25 of class II this is the case for only five of these. The six of class I and I-II have central surface brightness of $20.6 \pm 0.7 \mathrm{~B}-\mathrm{mag} \mathrm{arcsec}^{-2}$, and the five of class II $21.2 \pm 1.0$. For the Galaxy this parameter is $21.1 \pm 0.3$. In view of these limited statistics, I conclude that the Galaxy is probably not of luminosity class I but is likely I-II or II. So we appear to live in an SbI-II galaxy, and these are among the largest spirals in the universe.

\section{THE MILKY WAY COMPARED WITH OTHER GALAXIES}

We have seen that we live in a relatively large spiral galaxy of intermediate Hubble type. I have already indicated that $10 \%$ of all disk stars in the universe live in spirals with a disk scale length larger than $4 \mathrm{kpc}$. In this final section I will discuss the question of the Galaxy's appearance in relation to known nearby galaxies. In the semipopular literature and fundamental textbooks, one often sees M31 or M81 illustrated as galaxies looking much like our own.

De Vaucouleurs (see e.g., de Vaucouleurs and Pence 1978) has used various arguments through the years to identify systems that would bear a close resemblance. De Vaucouleurs and Pence have concluded on this basis that the morphological classification is SAB(rs)bcII and have published a sketch of what this morphology implies for the outsider's view. This is partly based on known morphological features, mainly from $\mathrm{H}$ I distributions and the distribution of $\mathrm{H}$ II regions, such as the definition and pitch angle of the spiral arms and the possible presence of a central bar and ring.

I have searched the literature for galaxies that are similar to our own in some parameters but that are not governed by the morphology of the spiral structure. These are a disk photometric scale length (which is Hubble constant-dependent) of 4 to $6 \mathrm{kpc}$, a bulge luminosity of about $10-20 \%$ of the total, a bulge effective radius of $2-3 \mathrm{kpc}$, a disk color (B-V) of $0.6-0.8$, a rotational velocity (in the flat part) of $210-230 \mathrm{~km} \mathrm{~s}^{-1}$, and an $\mathrm{H} \mathrm{I}$ content of a few times $10^{9} M_{\odot}$. Note that the disk luminosity is not a parameter on which restrictions are placed since the galaxies are supposed to conform to Freeman's law, and therefore this parameter is set by the scale length. Also, the total luminosity is restricted to lie within a range of $\sim 0.5 \mathrm{mag}$ of that predicted by the Tully-Fisher relation for galaxies within the narrow range of rotation velocities considered there. Finally, the Hubble type is somewhat re-stricted through the ratio of bulge-to-total luminosity and disk color. Of course, the number of galaxies for 
which all this information is available is not very large; it is mainly set by the requirement that full surface photometry is available, so that disk-bulge separation can be performed. The rotational velocity and $\mathrm{H}$ I content are known already from single-dish $21 \mathrm{~cm}$ line observations.

I have already indicated the resemblance of the Galaxy to NGC 891. This similarity cannot be positively stated from a survey as described since, for edge-on galaxies, all but the integrated disk luminosity and color are known. However, leaving these aside, NGC 891 does still come out as a close twin. Some galaxies that are often suggested in the literature as a close twin do not conform to these strict limits, if the limits are applied all at the same time. For example, M31 has too large a disk scale length, too bright a bulge, and too large a rotational velocity. M81 also rotates too fast (about $255 \mathrm{~km} \mathrm{~s}^{-1}$ ) and might have too small a scale length (2.5 kpc according to Kent 1987). NGC 4565 has too bright a bulge and also too high a rotational velocity (about $260 \mathrm{~km} \mathrm{~s}^{-1}$ ). Finally, the SbI galaxy NGC 3200 , which was proposed by Rubin (1983) to be similar to the Galaxy on the basis of its rotational curve, does not qualify on the basis of the disk scale length of about $8 \mathrm{kpc}$ on the distance scale used here (Kent 1986).

TABLE 1. Comparison of Parameters for the Milky Way, NGC 891, and NGC 5033

\begin{tabular}{llll}
\hline \hline & Galaxy & NGC 891 & NGC 5033 \\
\hline Distance $(\mathrm{Mpc})$ & & 9.5 & 14 \\
Bulge: & & & \\
$R_{\mathrm{e}}(\mathrm{kpc})$ & 2.7 & 2.3 & 2.9 \\
$\quad$ b/a & 0.7 & 0.7 & $?$ \\
$L_{\text {bulge }}\left(L_{\mathrm{B}, \odot}\right)$ & $2 \times 10^{9}$ & $1.5 \times 10^{9}$ & $4 \times 10^{9}$ \\
Disk: & & & \\
$\mu_{0}\left(\mathrm{~B}-\mathrm{mag} \operatorname{arcsec}^{-2}\right)$ & 22.1 & 22.9 (old disk) & 22.0 \\
$h(\mathrm{kpc})$ & 5 & 4.9 & 5 \\
$L_{\text {disk }}\left(L_{\mathrm{B}, \odot}\right)$ & $1.7 \times 10^{10}$ & $6.7 \times 10^{9}$ (old disk) & $1.7 \times 10^{10}$ \\
$(\mathrm{~B}-\mathrm{V})$ & 0.8 & 0.9 (old disk) & 0.6 \\
$R_{\max }(\mathrm{kpc})$ & $20-25$ & 21 & 22 \\
$L_{\text {bulge }} L_{\text {tot }}$ & 0.12 & 0.07 & 0.19 \\
$V_{\text {rot }}\left(\mathrm{km} \mathrm{s}^{-1}\right)$ & 220 & 225 & 215 \\
$M_{\mathrm{HI}}\left(M_{\odot}\right)$ & $8 \times 10^{9}$ & $4 \times 10^{9}$ & $4 \times 10^{9}$ \\
\hline
\end{tabular}

The only galaxy that does come close in all these respects is the Sbc(s)I-II spiral NGC 5033. Photometry of this galaxy has been given by Wevers, van der Kruit, and Allen (1986) and Kent (1987), and a picture of this galaxy appears in the first of these references. The assumed distance is $14 \mathrm{Mpc}$. The disk is not entirely exponential, but the profiles from these studies can be represented reasonably well with the parameters $R_{\mathrm{e}}=2.9 \mathrm{kpc}$ and, for a color $(B-V)=0.9$ and a flattening of 0.5 , luminosity $4 \times 10^{9} L_{\mathrm{B}, \odot}$ for the bulge and for the disk $\mu_{0}=$ 22.0 B-mag $\operatorname{arcsec}^{-2}$ and $h=5 \mathrm{kpc}$. Some parameters for the three galaxies are given in Table 1. For NGC 891 the disk parameters apply only to the old disk population, and for the bulgeto-total luminosity ratio it has been assumed that the younger populations provide as much Blight as the old disk.

It is interesting to note that NGC 5033 has a somewhat brighter bulge and lower H I mass than the Galaxy; on this basis the latter would be of somewhat earlier Hubble type than Sbc. This offsets the comparison to M31, which would make the Galaxy of slightly later type than Andromeda (Sb). 


\section{REFERENCES}

Arp, H. C. 1965, Ap. J., 142, 402.

Bahcall, J. N. and Soniera, R. M. 1984, Ap. J. Suppl., 55, 67.

Blitz, L., Fich, M., and Kulkarni, R. M. 1983, Science, 46, 177.

Chromey, F. R. 1979, Astron. J., 84, 534.

de Vaucouleurs, G. 1948, Ann. d'Astrophys., 11, 247.

de Vaucouleurs, G. 1959, Handbuch der Physik, 53, 511.

de Vaucouleurs, G. 1979, Observatory, 99, 128.

de Vaucouleurs, G. and Pence, W. D. 1978, Astron. J., 83, 1163.

Disney, M. J. 1976, Nature, 263, 573.

Disney, M. J. and Phillipps, S. 1983, MNRAS, 205, 1253.

Freeman, K. C. 1970, Ap. J., 160, 811.

Freeman, K. C. 1987, Ann. Rev. Astron. Astrophys., 25, 603.

Gilmore, G. and Reid, N. 1983, MNRAS, 202, 33.

Gilmore, G., Wyse, R. F. G., and Kuijken, K. 1989, Ann. Rev. Astron. Astrophys., 27 (in press).

Grosbol, P. J. 1985, Astron. Astrophys. Suppl., 60, 261.

Herschel, W. 1785, Phil. Trans., LXXV, 213.

Kent, S. M. 1986, Astron. J., 91, 1301.

Kent, S. M. 1987, Astron. J., 93, 816.

King, I. R. 1976, The Universe Unfolding (San Francisco: Freeman).

Kormendy, J. and Illingworth, G. 1982, Ap. J., 256, 460.

Lewis, J. R. 1986, Ph. D. thesis, Australian Nat. Univ.

Lewis, J. R. and Freeman, K. C. 1989, Astron. J., 97, 139.

Patterson, F. S. 1940, Harvard Bull. No. 914, p. 9.

Plaut, L. 1965, Stars and Stellar Systems V, ed. A. Blaauw and M. Schmidt (Univ. of Chicago Press), p. 267.

Roach, F. E. and Megill, L. R. 1961, Ap. J., 133, 228.

Rubin, V. C. 1983, in Kinematics, Dynamics, and Structure of Galaxies, ed. W. L. H. Schuter (Dordrecht: Reidel), p. 379.

Toller, G. 1987, in Proc. IAU 139, Galactic and Extragalactic Background Radiation, ed. S. Bowyer and C. Leinert, (Dordrecht: Kluwer Academic Publisher).

van der Kruit, P. C. 1979, Astron. Astrophys. Suppl., 38, 15.

van der Kruit, P. C. 1984, Astron. Astrophys., 140, 470.

van der Kruit, P. C. 1986, Astron. Astrophys., 157, 230.

van der Kruit, P. C. $1987 a$, Astron. Astrophys., 173, 59.

van der Kruit, P. C. 1987b, in The Galaxy, ed. G. Gilmore and B. Carswell (Dordrecht: Reidel), p. 27.

van der Kruit, P. C. and Freeman, K. C. 1986, Ap. J., 30, 556.

van der Kruit, P. C. 1988, Astron. Astrophys., 192, 117.

van der Kruit, P. C. and Searle, L. 1981a, Astron. Astrophys., 95, 105.

van der Kruit, P. C. and Searle, L. 1981b, Astron. Astrophys., 95, 116.

van der Kruit, P. C. and Searle, L. 1982a, Astron. Astrophys., 110, 61.

van der Kruit, P. C. and Searle, L. 1982b, Astron. Astrophys., 110, 79.

van Rhijn, P. J. 1925, Publ. Groningen Astron., Obs. No. 43.

Walterbos, R. and Kennicutt, R. C. 1987, Astron. Astrophys., 198, 61.

Watanbe, H. 1983, Annals Tokyo Obs., 2nd Ser., 19, 121.

Wevers, B. M. H. R. 1984, Ph. D. thesis, Univ. of Groningen.

Wevers, B. M. H. R., van der Kruit, P. C., and Allen, R. J. 1986, Astron. Astrophys. Suppl., 66, 505.

Wielen, R. 1977, Astron. Astrophys., 60, 263.

Young, J. S. 1987, Star Formation in Galaxies, NASA Conf. Proc., 2466, 197.

M. Longair: We have heard values for the disc scale length of the galaxy of 5-5.5 kpc from the optical data and $4 \mathrm{kpc}$ from the infrared data. Are the optical and infrared astronomers satisfied with the results?

P. Van der Kruit: I think we can agree that a value of about $4.5 \mathrm{kpc}$ is within the uncertainties of all determinations. 